\title{
Eletroacupuntura na anestesia com propofol em cães
}

\author{
Electroacupuncture on propofol anaesthesia in dogs
}

\author{
Renata Navarro Cassu' ${ }^{I}$ Dulce Mara Machado Pessoa ${ }^{\text {II }}$ \\ Stelio Pacca Loureiro Luna ${ }^{\text {III }}$
}

\section{RESUMO}

Resultados satisfatórios têm sido relatados com o emprego da eletroacupuntura (EA), como adjuvante da anestesia geral no homem e em animais. O objetivo do trabalho é avaliar a dose de indução anestésica do propofol em função do emprego da eletroacupuntura em cães. Foram utilizados 20 cães, distribuídos em dois grupos de igual número, GEA: foi realizada EA nos acupontos estômago 36 (E36), vesícula biliar 34 (VB 34) e baço-pâncreas 6 (BP 6), bilateralmente, durante 45 minutos antes da indução anestésica e GC: não foi realizada EA antes da indução anestésica. Os animais foram tranqüilizados com acepromazina intravenosa $\left(0,05 \mathrm{mg} . \mathrm{kg}^{-1}\right)$ 60 minutos antes da indução anestésica, realizada com propofol na taxa de 0,2ml.kg.min ${ }^{-1}$. A análise estatística foi realizada por test $t$ não pareado $(P<0,05)$. Os valores foram apresentados em média $\pm S D$. Não houve diferença significativa na dose do propofol entre os grupos $\left(5 \pm 2 \mathrm{mg} \mathrm{kg}^{-1}\right.$ no GC $e$ $5,2 \pm 1,6 \mathrm{mg} \mathrm{kg}^{-1}$ no GEA), sugerindo que a eletroacupuntura não potencializou o efeito depressor do propofol sobre o sistema nervoso central.

Palavras-chave: eletroacupuntura, propofol, cão.

\section{ABSTRACT}

Electro-acupuncture (EA) has been used as an adjuvant of general anesthesia in men and animals. The aim of this study was to evaluate the effect of EA on propofol dose for induction of anesthesia in dogs. Twenty healthy adult crossbred dogs were used and randomly distributed in two groups (n=10 per group): GEA dogs were submitted to EA in the acupoints stomach 36 , gall bladder 34 and spleen 6 , bilaterally, for 45 minutes before induction of anesthesia. Dogs in the control group (GC) were not treated before induction of

\begin{abstract}
anesthesia. Animals were sedated with $0.05 \mathrm{mg} \mathrm{kg}^{-1}$ of acepromazine intravenously. Anesthesia was induced with intravenous propofol using a $0.2 \mathrm{ml} . \mathrm{kg} \mathrm{min}^{-1}$ infusion rate 60 minutes after sedation. The statistical analysis was accomplished by the unpaired t test to compare differences between groups $(P<0.05)$. Values are presented as mean $\pm S D$. There was no significant difference in the dose of propofol required to abolish the podal withdrawal reflex between groups $\left(5.0 \pm 2.0 \mathrm{mg} \mathrm{kg}^{-1}\right.$ for the control group and $5.2 \pm 1.6 \mathrm{mg} \mathrm{kg}^{-1}$ for the EA treated group), suggesting that EA did not potentiate propofol anesthesia in dogs.
\end{abstract}

Key words: electroacupuncture, propofol, dog.

\section{INTRODUÇÃO}

A acupuntura tem sido extensamente empregada na China desde a década de 50 para analgesia cirúrgica. No entanto, a acupuntura isolada para realização de procedimentos cirúrgicos não suprime totalmente os estímulos nociceptivos, além de induzir pobre relaxamento muscular, sem atenuação dos reflexos autônomos induzidos pela tração visceral, em casos de cirurgias abdominais (SIM et al., 2002). Dessa forma, a acupuntura tem sido utilizada com sucesso quando associada às técnicas anestésicas convencionais, de modo a permitir a redução da dose dos anestésicos e analgésicos utilizados nos períodos intra e pós-operatórios, determinando maior conforto

'Departamento de Cirurgia e Anestesiologia Veterinária, Faculdade de Ciências Agrárias, Universidade do Oeste Paulista (Unoeste), Rodovia Raposo Tavares, Km 572, Campus II, Bairro Limoeiro, 19067-175, Presidente Prudente, SP, Brasil. E-mail: renavarro@uol.com.br. Autor para correspondência.

${ }^{\text {IIC } C u r s o ~ d e ~ M e d i c i n a ~ V e t e r i n a ́ r i a, ~ U n o e s t e, ~ P r e s i d e n t e ~ P r u d e n t e, ~ S P, ~ B r a s i l . ~}$

IIIDepartamento de Cirurgia e Anestesiologia Veterinária, Universidade Estadual Paulista (Unesp), Campus de Botucatu, Botucatu, SP, Brasil. 
ao paciente em relação ao uso dos fármacos isolados (KHO \& ROBERTSON, 1997; KOTANI et al., 2001). A redução do requerimento de anestésicos voláteis e opióides durante o procedimento anestésico é um fator de grande relevância clínica, pois permite a minimização dos efeitos depressores dos fármacos, além de favorecer a recuperação pós-anestésica. Estudos realizados no homem relataram resultados satisfatórios, com redução de $11 \%$ no requerimento do anestésico geral inalatório com o emprego de estímulo elétrico de pontos auriculares (GREIF et al., 2002) e 8\% com o uso de acupuntura manual nos mesmos pontos (TAGUCHI et al., 2002). Porém, resultados divergentes foram relatados por MORIOKA et al. (2002), com o estímulo dos pontos Zusanli, Yanglingquan e Kunlun.

O objetivo deste estudo foi avaliar a dose de indução anestésica com propofol em cães saudáveis com o uso prévio de eletroacupuntura.

\section{MATERIAL E MÉTODOS}

Foram utilizados 20 cães, clinicamente saudáveis (avaliados por exame físico e exames laboratoriais, incluindo hemograma, função hepática e função renal), fêmeas ( $n=12)$ e machos $(n=8)$, sem raça definida, adultos, com peso médio de $7,5 \pm 2 \mathrm{~kg}$, provenientes do canil da instituição de origem.

Em experimento duplo-cego, os animais foram distribuídos em dois grupos de igual número $(n=10)$, denominados de GEA e GC. Todos os animais foram tranqüilizados com $0,05 \mathrm{mg} \mathrm{kg}^{-1}$ de maleato de acepromazina ${ }^{\mathrm{b}}$, pela via intravenosa ,e após 15 minutos foram posicionados em decúbito lateral esquerdo. Nos animais do GEA, foi realizada eletroacupuntura nos acupontos: estômago 36 (E 36), vesícula biliar 34 (VB 34) e baço-pâncreas 6 (BP 6), bilateralmente, durante 45 minutos antes da indução anestésica. Foi utilizado estímulo elétrico com freqüência modo denso/ disperso $(10-300 \mathrm{~Hz})$, onda quadrada e corrente alternada. Os pontos VB 34 e BP 6 foram estimulados pela mesma fonte, enquanto que o ponto E 36 do lado direito foi estimulado pela mesma fonte usada para estimular o E 36 do lado esquerdo. O ponto E36 (Zusanli) está localizado aproximadamente $5 \mathrm{~cm}$ distal à cabeça da fíbula, lateral à crista da face lateral da tíbia. O ponto VB34 (Yang-ling-chuan) está localizado na junção proximal entre a tíbia e a fíbula, entre o músculo tibial cranial e o músculo peroneu longo. O ponto BP6 (San-yin-chiao) está localizado 3cm proximal ao maléolo medial, na borda caudal da tíbia, na altura do cruzamento entre a veia safena e a borda caudal da tíbia (Figuras 1 e 2). As agulhas foram introduzidas nos referidos pontos, com adaptação dos eletrodos no corpo das mesmas, com o aparelho de eletroacupuntura ${ }^{c}$ desligado. Iniciou-se com corrente zero e elevou-se a mesma lentamente, até que o animal respondesse demonstrando desconforto, momento em que a intensidade foi levemente reduzida, mantendose o estímulo suficiente para observação de contrações musculares localizadas. A EA foi realizada durante 45 minutos. No GC (grupo controle) utilizou-se o mesmo protocolo de GEA, excetuando-se a EA.

Em ambos os grupos, a anestesia foi induzida 60 minutos após a aplicação da acepromazina, com propofol $^{\mathrm{d}}$, mediante bomba de infusão ${ }^{\mathrm{e}}$, na taxa de

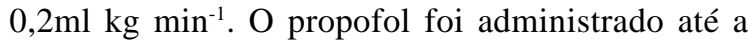
obtenção da ausência do reflexo interdigital, aferido no membro anterior direito entre o $2^{\circ}$ e 3 o dígitos, com estímulo nociceptivo mecânico, realizado com pinça hemostática Kelly, com ranhuras protegidas (LUNA et al., 2002). A dose e o tempo necessários para a obtenção de ausência de reflexo interdigital foram registrados em $\mathrm{mg} \mathrm{kg}^{-1}$ e segundos, respectivamente. Os valores foram expressos pela média \pm SD.

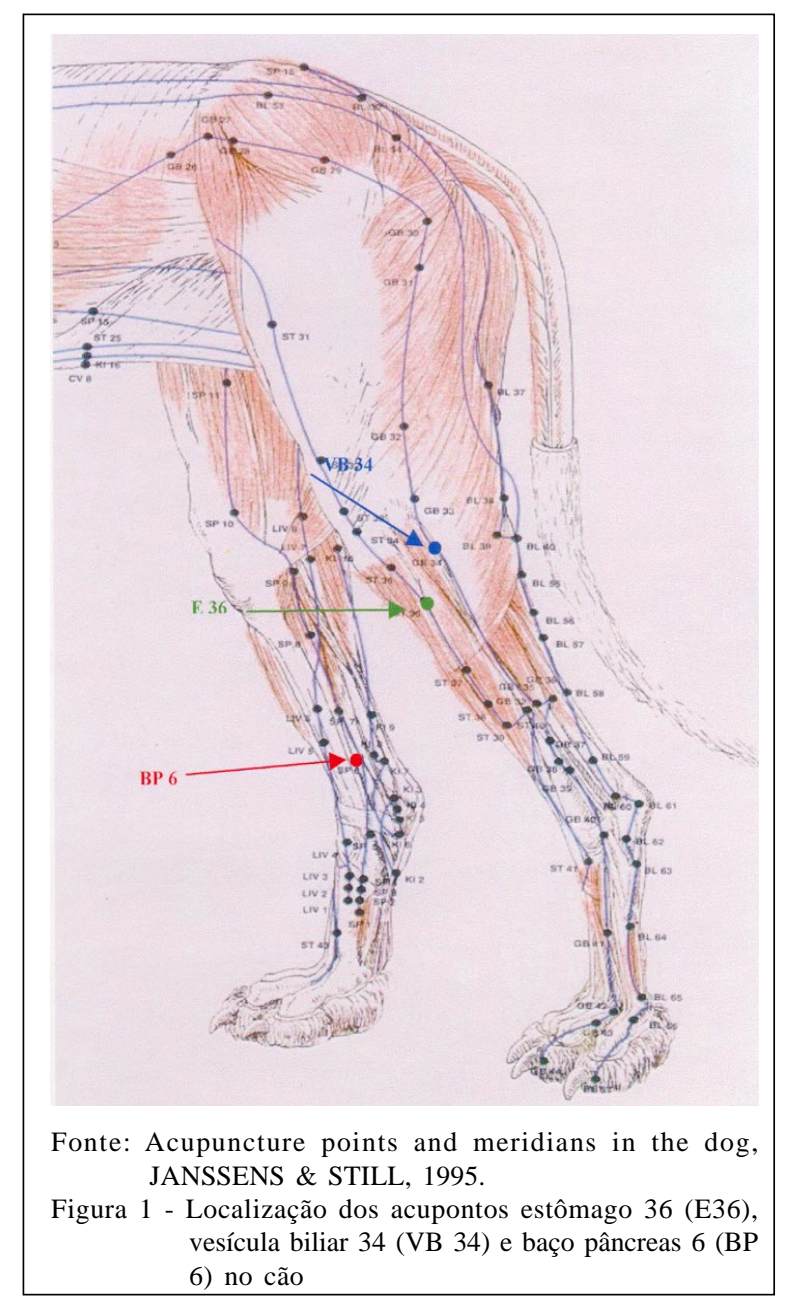

Ciência Rural, v.38, n.6, set, 2008. 


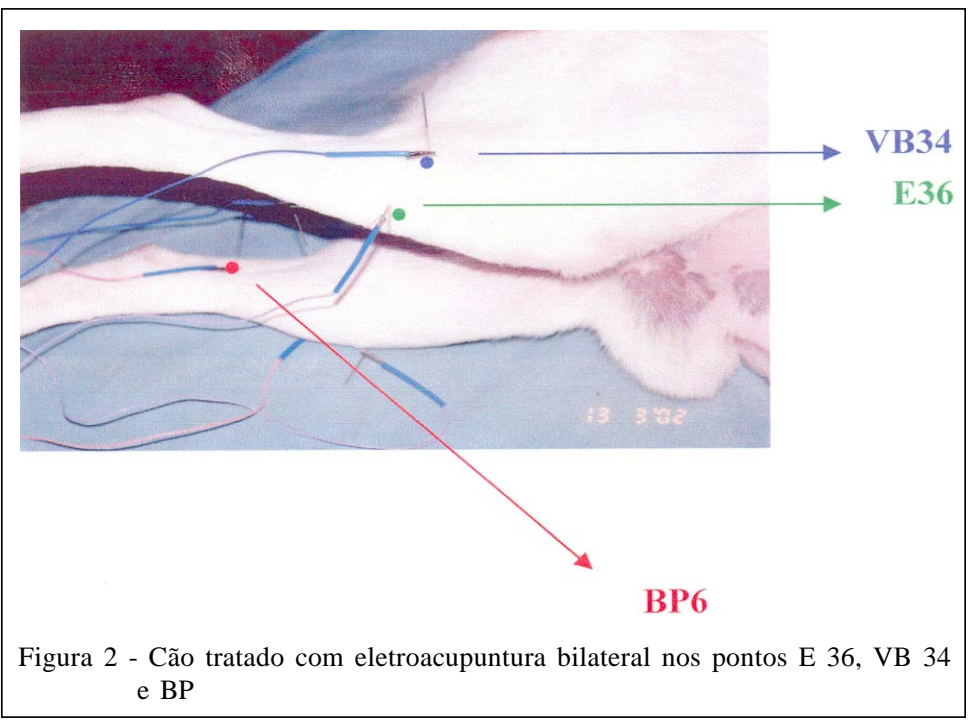

esses resultados não foram relevantes, pois a administração sistêmica de opióides reduz o requerimento transoperatório de anestésicos voláteis ou injetáveis, entre 20 a 60\% (MURPHY \& HUG, 1982; VALVERDE et al., 1989; GEEL, 1991).

A divergência entre os resultados observados pode estar vinculada à metodologia utilizada por cada pesquisador. Os efeitos da acupuntura podem variar em função dos pontos selecionados, das propriedades e do estímulo elétrico empregado (MEISSNER et al., 2004). Os acupontos estimulados no presente estudo, E36, VB34 e BP6, são

A análise estatística foi realizada por meio de teste t não pareado, sendo considerado nível de 5\% de significância $(\mathrm{P}<0,05)(\mathrm{ZAR}, 1996)$.

\section{RESULTADOS}

Não foram observadas diferenças significativas nas doses de indução anestésica do propofol, com dose média de $5 \pm 2 \mathrm{mg} \mathrm{kg}^{-1}$ para o GC e 5,2 $\pm 1,6 \mathrm{mg} \mathrm{kg}^{-1}$ para o GEA. O tempo necessário para a obtenção da anestesia não diferiu entre os grupos, variando entre $200 \pm 2,4$ e $263 \pm 2$ segundos para o GEA e $\mathrm{GC}$, respectivamente.

\section{DISCUSSÃO}

No presente estudo, a dose média de propofol necessária para a indução anestésica ficou dentro dos limites relatados para a espécie canina, que estão compreendidos entre 2,5 a 5,5 $\mathrm{mg} \mathrm{kg}^{-1}$, após o uso de medicação pré-anestésica (HALL \& CHAMBERS, 1987; WATNEY \& PABLO, 1992). Porém, não foi observada redução na dose anestésica do propofol após o uso da EA, corroborando resultados relatados no homem, em estudo similar, no qual a dose de manutenção anestésica do propofol não foi alterada pela aplicação da EA nos acupontos E36, BP6 e F3 (MEISSNER et al., 2004). Resultados divergentes foram relatados em cães, com redução de $10 \%$ no requerimento anestésico em função do estímulo elétrico dos pontos IG4, P7, VG 14 e 20, San Tai e Baihui (CULP et al., 2005). No homem, foram observadas reduções de 8,5\% a $11 \%$ no requerimento anestésico transoperatório com a aplicação de acupuntura em pontos auriculares (GREIF et al., 2002; TAGUCHI et al., 2002). Porém, clinicamente, mediadores de efeito analgésico pronunciado, conforme relatado em estudos prévios (O’BOYLE \& VAJDA, 1975; WRIGHT \& MCGRATH,1981, CASSU et al., 2008). $\mathrm{O}$ interesse pelo emprego de pontos indutores de analgesia está relacionado ao mecanismo pelo qual a EA permite a redução do requerimento dos anestésicos gerais. O estímulo desses acupontos promove a ativação de determinados receptores no corno dorsal da medula espinhal, culminando com o aumento de opióides endógenos, como as endorfinas e as encefalinas, de modo a potencializar a depressão central induzida pelo anestésico geral (MEISSNER et al., 2004). Paralelamente, outros neurotransmissores também estão envolvidos com os mecanismos de ação da acupuntura, destacando-se: a acetilcolina, que atua facilitando o efeito analgésico, as catecolaminas (norepinefrina e dopamina), serotonina e o ácido gamaaminobutírico(GABA) (KLIDE \& GAYNOR, 2001). Com relação à escolha do estímulo elétrico, o modo densodisperso, com freqüência mista, variando entre 10-300 $\mathrm{Hz}$, utilizado neste estudo, favorece a liberação de diferentes opióides endógenos como as endorfinas, as encefalinas e as dinorfinas, contribuindo no efeito analgésico mediado pela acupuntura (HSU, 1996).

Contudo, apesar de estudos prévios terem relatado efeito analgésico pronunciado com o emprego dos acupontos e do estímulo elétrico selecionados neste estudo, não foi possível a detecção de sinergismo entre a EA e o anestésico geral propofol.

\section{CONCLUSÃO}

Diante das condições experimentais deste estudo, pode-se concluir que a aplicação de EA nos pontos E36, BP6 e VB34 previamente à indução

Ciência Rural, v.38, n.6, set, 2008. 
anestésica não favorece a redução da dose do agente indutor em cães, sugerindo-se que esta técnica não se mostra satisfatória para potencializar o efeito depressor central induzido pelo propofol.

\section{FONTES DE AQUISIÇÃO}

agulhas de acupuntura descartáveis, Suzhou Huanqiu, Acupuncture Medical Applance, CO, Ltda, China.

${ }^{\mathrm{b}}$ Acepran 0,2\%, Univet, Brasil.

'Sikuro, DS100, Brasil.

dPropovan, Cristália, Brasil.

eLifeMed, Fars 600, Brasil.

\section{COMITÊ DE ÉTICA}

Este estudo foi aprovado pelo Comitê de Ética da instituição de origem, sob o protocolo n.012/05.

\section{REFERÊNCIAS}

CASSU, R.N. et al. Electroacupuncture analgesia in dogs: is there a difference between uni or bilateral stimulation? Veterinary Anaesthesia and Analgesia, v.35 p.52-61, 2008.

CULP, L.B. et al. Comparisons of the effects of acupuncture, electroacupuncture, and transcutaneous cranial electrical stimulation on the minimum alveolar concentration of isoflurane in dogs. American Journal Veterinary Research, v.66, n.8, p.1364-1370, 2005.

GEEL, J.K. The effect of premedication on the induction dose of propofol in dogs and cats. Journal of the South African Veterinary Association, v.62, p.118-123, 1991.

GREIF, R. et al. Transcutaneous electrical stimulation of an auricular acupuncture point decreases anesthetic requirement. Anesthesiology, v.96, p.306-312, 2002.

HALL, L.W.; CHAMBERS, J.P. A clinical trial of propofol infusion anesthesia in dogs. Journal of Small Animal Practice, v.28, p.623-637, 1987.

HSU, D.T. Acupuncture: a review. Regional Anesthesia, v.21, p.361-370, 1996.

JANSSENS, L.; STILL, J. Acupuncture points and meridians in the dog. Van Wilderode: Zaventem, 1995. 14p.

KHO, H.G.; ROBERTSON, E.N. The mechanisms of acupuncture analgesia: review and update. American Journal of Acupuncture, v.25, p.261-281, 1997.
KLIDE, A.M.; GAYNOR, J.S. Acupuncture for surgical analgesia and postoperative analgesia. In: SHOEN, A.M. Veterinary acupuncture: ancient art to modern medicine. 2.ed. St. Louis: Mosby, 2001. Cap.24, p.295-301.

KOTANI, N. et al. Preoperative intradermal acupuncture reduces postoperative pain, nausea and vomiting, analgesic requirement, and sympathoadrenal responses. Anesthesiology, v.95, 349-356, 2001.

LUNA et al. Effect of acupuncture on the thiopental dose for induction of anaesthesia in dogs. Revista Brasileira de Ciência Veterinária, v.9, p.286-288, 2002.

MEISSNER, et al. Acupuncture decreases somatosensory evoked potential amplitudes to noxious stimuli in anesthetized volunteers. Anesthesia and Analgesia, v.98, p.141-147, 2004.

MORIOKA, N. et al. Electro-acupuncture at the Zusanli, Yanglingquan and Kunlun points does not reduce anesthetic requirement. Anesthesia and Analgesia, v.95, p.98-102, 2002.

MURPHY, M.R.; HUG, C.C. The anesthetic potency of fentanyl in terms of its reduction of enflurane MAC. Anesthesiology, v.57, p.485-488, 1982.

O’BOYLE, M.A.; VAJDA, G.K. Acupuncture anesthesia for abdominal surgery. Modern Veterinary Practice, v.56, p.705707, 1975.

SIM, C.K., et al. Effects of electroacupuncture on intraoperative and postoperative analgesic requirement. Acupuncture in medicine: Journal of the British Medical Acupuncture Society, v.20, n.2-3, p.56-65, 2002.

TAGUCHI, A. et al. The effect of auricular acupuncture on anaesthesia with desflurane. Anaesthesia, v.57, p.1159-1163, 2002.

VALVERDE, A. et al. Epidural morphine reduces halothane MAC in the dog. Canadian Journal of Anaesthesia, v.36, p.629-632, 1989.

WATNEY, G.C.; PABLO, L.S. Median effective dosage of propofol for induction of anaesthesia in dogs. American Journal Veterinary Research, v.53, p.2320-2322, 1992.

WRIGHT, M.; McGRATH, C.J. Physiologic and analgesic effects of acupuncture in the dog. Journal of the American Veterinary Medical Association, v.178, p.502-507, 1981.

ZAR, J.H. Biostatistical analysis. New Jersey: PrenticeHall Upper Saddle River, 1996. p.718. 